\title{
Perfect outcome of kidney recipients with ureteral stenosis after treatment with open surgery under magnetic resonance urography localization
}

\author{
Guangjun Liu $^{1,2,3,4,5}$, Xuliang Wang ${ }^{1,2,3,4,5}$, Hongfeng Huang ${ }^{1,2,3,4,5}$, Rending Wang ${ }^{1,2,3,4,5}$, Wenhan Peng ${ }^{1,2,3,4,5}$, \\ Jianghua Chen ${ }^{1,2,3,4,5}$, Jianyong $W \mathbf{u}^{1,2,3,4,5} \wedge$
}

${ }^{1}$ Kidney Disease Center, the First Affiliated Hospital, College of Medicine, Zhejiang University, Hangzhou, China; ${ }^{2}$ Key Laboratory of Kidney Disease Prevention and Control Technology, Zhejiang Province, Hangzhou, China; ${ }^{3}$ National Key Clinical Department of Kidney Diseases, Hangzhou, China; ${ }^{4}$ Institute of Nephrology, Zhejiang University, Hangzhou, China; ${ }^{5}$ The Third Grade Laboratory under the National State, Administration of Traditional Chinese Medicine, Hangzhou, China

Contributions: (I) Conception and design: J Wu; (II) Administrative support: J Chen; (III) Provision of study materials or patients: H Huang, R Wang, W Peng; (IV) Collection and assembly of data: G Liu; (V) Data analysis and interpretation: X Wang; (VI) Manuscript writing: All authors; (VII) Final approval of manuscript: All authors.

Correspondence to: Jianyong Wu, MD. 79\# Qingchun Road, Hangzhou 310003, China. Email:wujianyong1964@zju.edu.cn.

Background: To evaluate the outcome of kidney recipients with ureteral stenosis after treatment with open surgery under magnetic resonance urography (MRU) localization.

Methods: We assessed 2,256 consecutive kidney transplant recipients between October 2010 and December 2018. Ureteral stenosis was detected by ultrasound, confirmed and positioned by Magnetic Resonance Urography. All patients underwent open ureteral reconstruction. The ureteral stenosis was located according to the location on the MRU during the operation. Surgical complications and recurrence rate were recorded in the stenosis group. Outcomes were compared with those of a matched control group of transplant recipients with no history of ureteric stenosis.

Results: The incidence of ureteral stenosis in our center was 3.1\% (70/2,256). Sixty-four cases (91.4\%) were confirmed to have distal stenosis and were reconstructed with ureterovesical re-implantation; six cases $(8.6 \%)$ were confirmed to have mid-distal stenosis and were subjected to ureteroureterostomy with the use of native ureter. The overall success rate was $100 \%$ and the graft function was salvaged in all cases. There was no recurrence of stenosis after a mean follow-up of $38.9 \pm 26.3$ months. The complication rate was $5.7 \%$. The 110-month graft survival and patient survival were not significantly different between the stenosis and control groups.

Conclusions: MRU is an effective method for non-invasive and accurate diagnosis of ureteral stenosis in kidney transplant recipients. Open ureteral reconstruction surgery under MRU localization for treatment of ureter stenosis after kidney transplantation had a high success rate, low recurrence rate and high safety.

Keywords: Ureteral stenosis; open ureteral reconstruction; interventional management; MRU localization; kidney transplantation

Submitted Nov 07, 2020. Accepted for publication Jan 15, 2021.

doi: $10.21037 /$ tau-20-1404

View this article at: http://dx.doi.org/10.21037/tau-20-1404

$\wedge$ ORCID: 0000-0003-0957-2630. 


\section{Introduction}

Major ureteral complications following renal transplantation are a significant source of morbidity and mortality, ureteral stenosis is one of the most common urologic complications after renal transplant and has been reported in $0.6-12.5 \%$ of kidney recipients (1-3). The majority of ureteral stenosis occur within 3 months post- transplantation and are located in the distal ureter (4-7). Ureteral stenosis is thought to result from inadequate preparation of the transplant ureter, ischemia of the distal ureter, technical issues during implantation, episodes of rejection, infection (human polyoma BK virus nephropathy) or an external compression by a lymphocele or hematoma (4-8).

The majority of ureteral stenosis require interventional management or open surgical re-construction. There are pros and cons in either endoscopic or surgical treatment. There is no consensus about the optimal treatment strategy for ureteral stenosis and there are no guidelines for the choice of interventional therapy or open surgery.

The adhesion caused by repeated operation, the location of ureteral stenosis segment and the possible damage of transplanted kidney and blood vessel were the main difficulties of the open reconstructive surgery. Magnetic resonance urography (MRU) can be used to diagnose ureteral obstruction and accurately locate the obstruction site. All the patients with ureteral stenosis in our center were relieved by open surgery under MRU localization. This study was to review the outcomes of kidney recipients with ureteral stenosis who had undergone open ureteral reconstruction under MRU localization and to compare the outcomes with those without ureteral stenosis.

We present the following article in accordance with the TREND reporting checklist (available at http://dx.doi. org/10.21037/tau-20-1404).

\section{Methods}

\section{Data collection}

This retrospective matched cohort study was undertaken at a tertiary teaching hospital that provides a comprehensive renal service, including treatment of kidney diseases, dialysis and kidney transplantation. All kidney transplants in this study came from DCD donation or living donation, and no executed prisoner was used as a donor. The donor grafts of DCD were donated to the Red Cross Society of Zhejiang Province and allocated to our center by the China Organ Transplant Response System. The study was conducted in accordance with the Declaration of Helsinki (as revised in 2013). The study was approved by the ethics committee of the First Affiliated Hospital, School of Medicine, Zhejiang University (NO. 2019-655: the registration number of ethics board) and individual consent for this retrospective analysis was waived. All patients who developed ureteral stenosis following renal transplantation and subsequently underwent small incision open surgical ureteral reconstruction under MRU localization between October 2010 and December 2018 were included in the stenosis group. Once the stenosis group was identified, a control group was selected from the remaining patients. The two groups were matched for age, sex, type of transplant (living donor or deceased donor), the year of kidney transplantation (to ensure that immunosuppressant therapy and surgical technique were similar), and presence of diabetes mellitus and hypertension factors known to influence both graft and patient survival. The donor and/or graft characteristics were collected included age, sex, cold ischemia time and number of arteries. Data regarding episodes of rejection and infection due to human polyoma BK virus were collated. Patients were followed up until December 2019.

\section{Immunosuppressive therapy and surgical technique}

During the study period, standard triple immunosuppression protocols were implemented including prednisolone, mycophenolate mofetil and tacrolimus or cyclosporine after induction therapy with baliximab or rabbit anti-thymocyte globulin (rATG). Renal transplant was undertaken by dedicated transplant surgeons with more than 10 years of experience. The standard technique of renal transplantation included an extraperitoneal approach through an iliac fossa incision and vascular anastomoses of the external iliac vein, external iliac artery or internal iliac artery (living-kidney transplantation). An extravesical technique of ureteral implantation was routinely used for ureteroneocystostomy according to the Lich-Gregoir ureterovesical reimplantation technique. A double-J stent was used to bridge the anastomosis to the bladder in most patients. This stent was routinely removed about 4 weeks after surgery under local anesthesia.

\section{Diagnosis of ureteral stenosis and emergency treatment}

We monitored serum creatinine (Scr) levels daily for the first 7 days after transplantation, followed by weekly for 3 months and then monthly during the 1 st year. Thereafter, Scr levels were measured every 1-3 months. Any increase in Scr levels was promptly investigated and treated. New- 

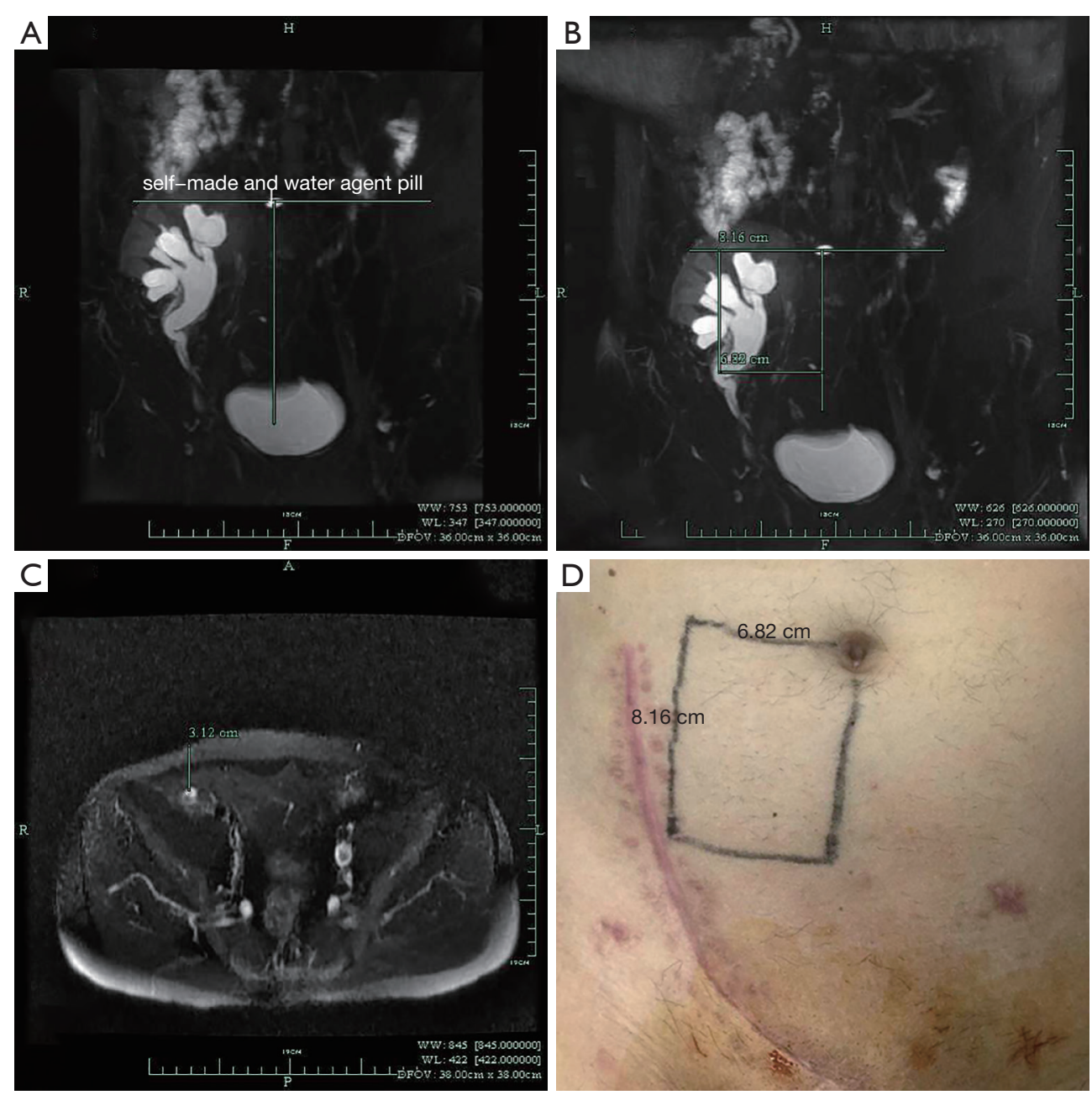

Figure $1 \mathrm{MRU}$ positioning method and body surface marking. A horizontal line was drawn at the umbilical level and a vertical line under the T2-weighted image (A). The distance from the horizontal line vertically downward to the upper end of the obstruction section and the horizontal distance from the vertical line to the upper segment of the obstruction were measured (B). The distance from the upper part of the obstruction to the skin surface was measured in cross section $(C)$. The upper point of the stenosis was marked on the body surface of the patient according to the measurements (D). MRU, magnetic resonance urography.

onset hydronephrosis of the transplanted kidney was detected by ultrasound and confirmed by MRU, and the location of the ureteral stenosis was recorded. If the patient just had an increase in Scr, there was no need to do any intervention before the MRU examination and surgery. If the patient developed hyperkalemia or heart failure, the patient will undergo hemodialysis treatment before the MRU examination and surgery.

\section{Technique of magnetic resonance urography localization}

Knowledge of the location and length of the stricture according to MRU was helpful for planning operation. The ureteral stenosis segment was well positioned in the MRU image and marked on the patient's body surface before surgery. One self-made and water-agent pill was placed at umbilicus before MRU examination for body surface positioning. We drew a horizontal line at the umbilical level and a vertical line under the T2-weighted image (Figure 1A), then measure the distance from the horizontal line vertically downward to the upper end of the obstruction section and the horizontal distance from the vertical line to the upper segment of the obstruction (Figure 1B). Finally, the distance from the upper part of the obstruction to the skin surface 

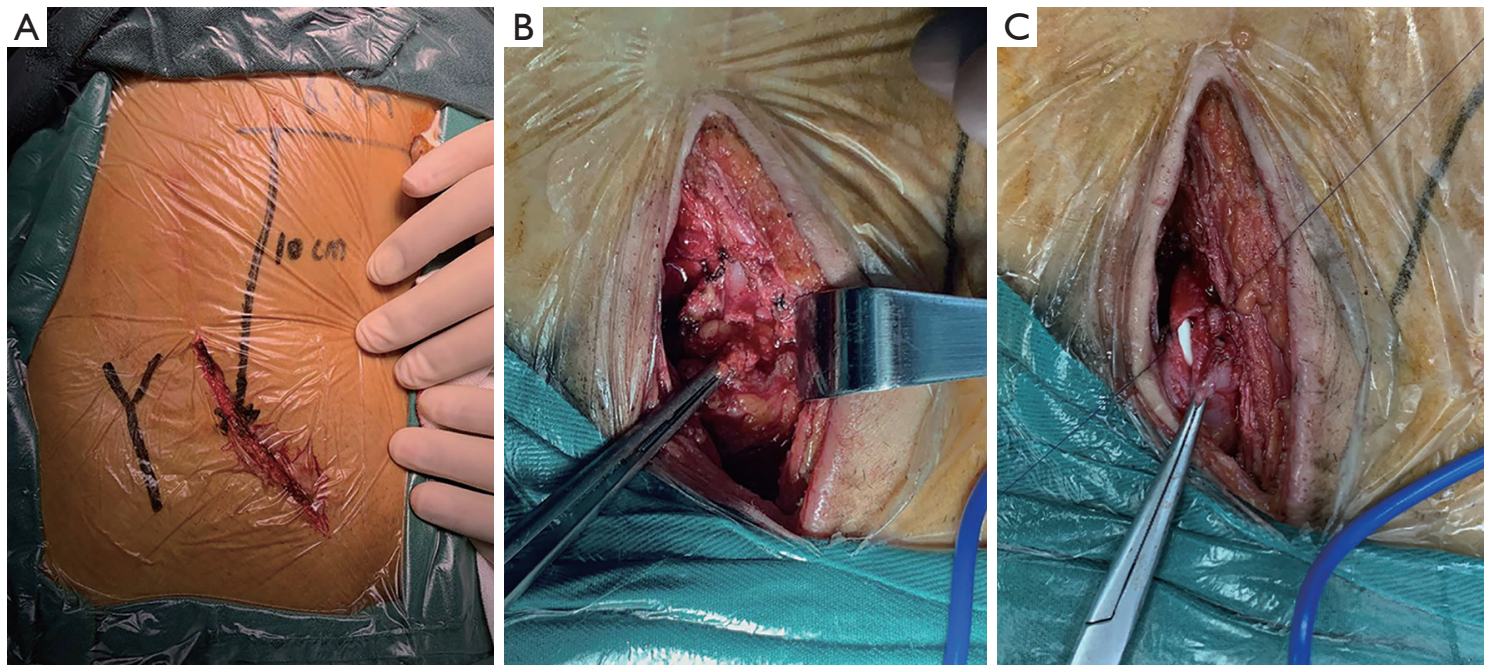

Figure 2 The procedure of open ureteral reconstruction surgery. The incision was made through the distal of the existing transplant scar (A). The strictured section of the ureter was excised and a small section of healthy ureter was isolated (B). A re-implantation technique was used over a double-J stent (C).

was measured in cross section (Figure 1C). According to the measurements, we marked the upper point of the stenosis on the body surface of the patient (Figure 1D). We measured the distance from the upper end of the stenosis to the anastomotic stoma of the bladder in MRU.

\section{Technique of open ureteral reconstruction}

Open ureteral reconstruction utilized an extraperitoneal approach in all cases. The incision was made through the distal of the existing transplant scar (Figure 2A). The strictured section of the ureter was further identified according to the location marked on the body surface. The stenosis was often difficult to identify and always in the most severe adhesion area. The strictured section of the ureter was excised and a small section of healthy ureter was isolated (Figure $2 B$ ). If the proximal nonstrictured healthy ureter was long enough to reach the bladder, a simple re-implantation technique was used over a double-J stent. This approach occasionally required mobilization of the urinary bladder and hitching stitches to the psoas muscle and adjacent adventitia to avoid tension on the anastomosis (Figure 2C). If re-implantation of the transplant ureter was not feasible, anastomosis to the native ureter was performed. The placement of double-J stent in the ipsilateral autogenous ureter before operation can help to locate the ureter during operation. Both reconstruction techniques need a double-J stent, which was removed after 4-6 weeks. The surgical complications were recorded according to Clavien-Dindo classification.

\section{Statistical analysis}

All data were analyzed by using SPSS 23 and STATA 16. Quantitative and qualitative variables were compared by using the $t$ test, McNemar test and Fisher's exact test. We evaluated graft and patient survival with the Kaplan-Meier method, and the groups were compared by using log-rank testing. $\mathrm{P}<0.05$ was considered statistically significant.

\section{Results}

Seventy patients developed a ureteral stricture that required intervention, yielding an overall incidence of $3.1 \%$. The incidence of living donor kidney transplantation was $3.8 \%$, while the incidence of DCD kidney transplantation was $2.6 \%$. A total of 70 patients were included in the stenosis group. The patient and graft characteristics of the two groups are listed in Tables 1 and 2. The underlying causes of end-stage renal disease, the number of rejection episodes, the incidence of delayed graft function (DGF), the development of human polyoma BK virus infection, and the graft characteristics, particularly intraoperative factors (cold ischemia time and vascular anatomy), were similar in the two groups.

The median time to develop stenosis was 3 months 
Table 1 Demographic and clinical data from patients with ureteral stenosis and matched control subjects

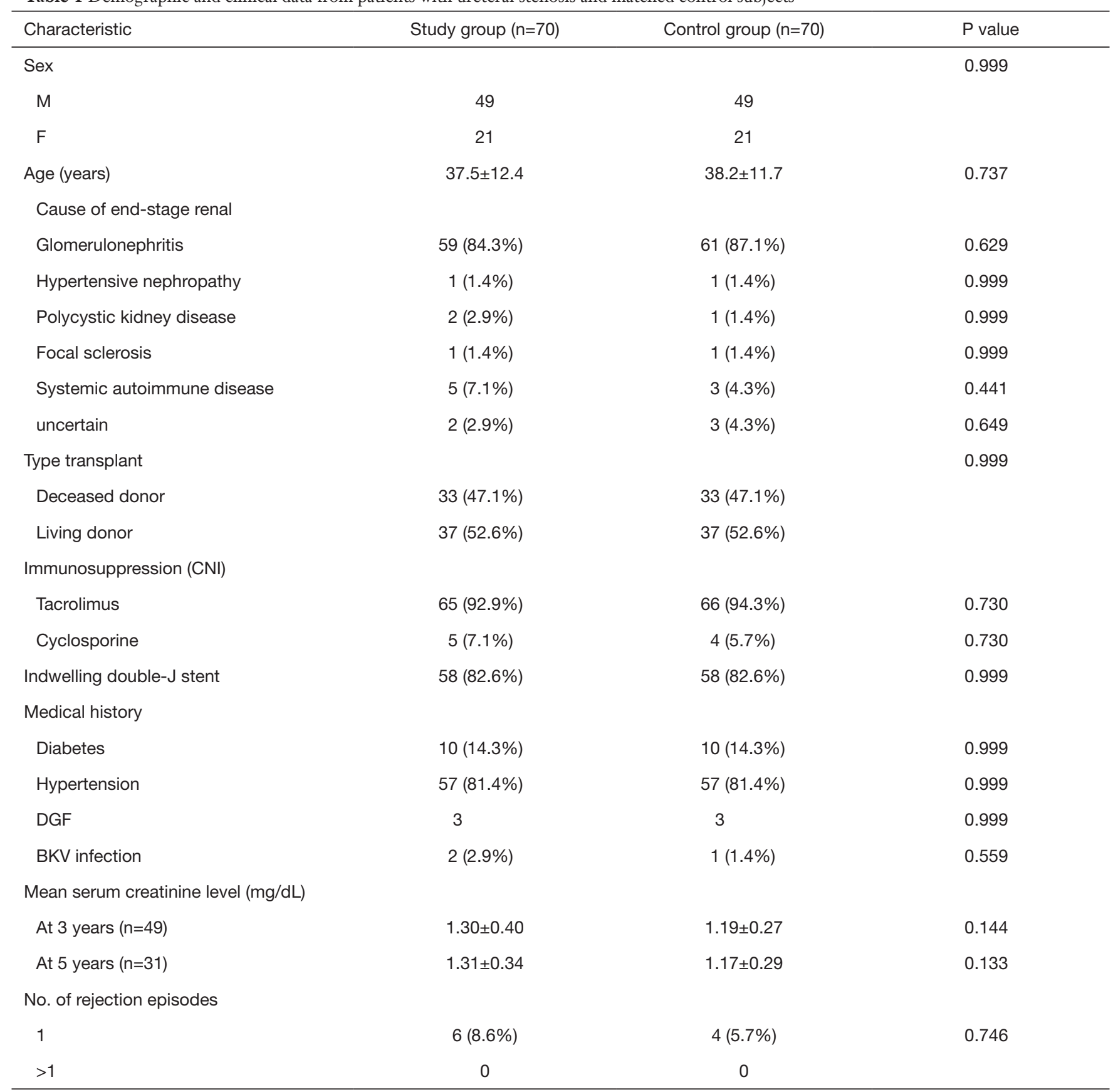

The two groups were matched for age, sex, history of diabetes mellitus and hypertension, and year of transplantation. DGF, delayed graft function; CNI, calcineurin Inhibitor; BKV, BK virus.

[range 1 week-36 months] from transplant, and the mean follow-up time was 43.6 months (range, 12-108 months). Ultrasonography showed that the mean hydronephrosis of the renal collecting system was $1.7 \pm 0.6 \mathrm{~cm}$. MRU showed 64 cases of distal and 6 mid-distal ureteral strictures. The average time from the diagnosis of the stenosis to the operation of the patients was $1.4 \pm 0.5$ days. All patients with ureteral stenosis were relieved by open surgery under MRU localization. The results seen during the operation are basically consistent with the results of MRU. Sixty- 
Table 2 Donor and graft characteristics of the study population

\begin{tabular}{|c|c|c|c|}
\hline Characteristic & Study Group $(n=70)$ & Control Group $(n=70)$ & $P$ value \\
\hline M & $41(58.6 \%)$ & 37 (52.9\%) & \\
\hline $\mathrm{F}$ & $29(41.4 \%)$ & $33(47.1 \%)$ & \\
\hline Mean age of donor (years) & $48.4 \pm 10.7$ & $46.7 \pm 12.8$ & 0.409 \\
\hline Deceased donor $(n=33)$ & $6.1 \pm 2.5$ & $6.9 \pm 2.5$ & 0.199 \\
\hline Living donor $(\mathrm{n}=37)$ & $1.9 \pm 0.8$ & $2.1 \pm 0.9$ & 0.333 \\
\hline No. of arteries & & & 0.353 \\
\hline One artery & 61 & 57 & \\
\hline
\end{tabular}

four cases $(91.4 \%)$ with distal ureteral stricture were reconstructed by re-implantation of the ureter to the bladder, and six cases (8.6\%) with mid-distal stenosis underwent anastomosis to the native ureter (Figure 3). The overall success was $100 \%$, with graft function being salvaged in all cases and with no stricture recurrence after a mean follow-up of $38.9 \pm 26.3$ months.

\section{Allograft function}

In stenosis group, the Scr was significantly elevated after ureteral stenosis $(1.32 \pm 0.42 \mathrm{mg} / \mathrm{dL}$ before, $3.92 \pm 2.98 \mathrm{mg} / \mathrm{dL}$ peak post ureteral stenosis, $\mathrm{P}<0.01)$. One week after the reconstruction of the ureter, the Scr was quickly decreased to $1.39 \pm 0.38 \mathrm{mg} / \mathrm{dL}, 1$ year later the mean $S c r$ was $1.44 \pm 0.52 \mathrm{mg} / \mathrm{dL}(\mathrm{P}>0.05$, compared with Scr before ureteral stenosis).

In stenosis group, there were 49 patients with a 3 -year follow-up and 31 patients with a 5 -year follow-up after the reconstruction of the stenosis. The mean Scr of 3 years and 5 years after the transplantation was $1.30 \pm 0.340$ and $1.31 \pm 0.34 \mathrm{mg} / \mathrm{dL}$, and in control group, the mean Scr of 3 years and 5 years after the transplantation was $1.19 \pm 0.27$ and $1.17 \pm 0.29 \mathrm{mg} / \mathrm{dL}$, and there were no significantly differences between two groups $(\mathrm{P}>0.05)$.

\section{Surgical time and complications}

The reconstructed open surgeries under MRU localization were undertaken by the same experienced transplant surgeon. The mean surgical time was $129.9 \pm 45.4 \mathrm{~min}$. The total complication rate was $5.7 \%(4 / 70)$, and there was no perioperative mortality (Table 3). Two patients suffered acute kidney injury after the operation. The renal vein was injured in one patient in the process of separating the ureter (NO.5), and renal function was impaired due to long-term interruption when repairing the renal vein. After one week of dialysis, urine volume increased to about $2,000 \mathrm{~mL}$ per day and the Scr gradually decreased to $1.46 \mathrm{mg} / \mathrm{dL}$. A $6.1 \times 3.0 \mathrm{~cm}$ subcapsular hematoma of the transplanted kidney occurred in one patient (NO. 19), and the Scr level increased from 1.45 to $4.2 \mathrm{mg} / \mathrm{dL}$. Without special treatment, the Scr gradually decreased to $1.42 \mathrm{mg} / \mathrm{dL}$ within 2 weeks and the subcapsular hematoma was gradually absorbed in one month. Urinary leakage occurred in 2 patients (NO. 50 and NO. 61) after reimplantation of the ureter to the bladder. It was successfully alleviated by urinary catheterization and the urine effusion drainage within 2 weeks. The two patients were on dialysis for more than 8 years and both had diabetes.

All patients with ureteral stenosis routinely undergo ultrasonography after the ureteral reconstruction. Five patients still existed a mild hydronephrosis of the renal collecting system $(0.76 \pm 0.19 \mathrm{~cm})$ after surgery, however, the Scr had dropped from $2.20 \pm 0.95$ to $1.23 \pm 0.27 \mathrm{mg} / \mathrm{dL}$. Native kidney hydronephrosis $(2.36 \pm 0.36 \mathrm{~cm})$ occurred in 5 of 6 patients who had anastomosis with native ureter, they all had no symptoms and no infection.

\section{Allograft and patient survival}

There was no graft loss or patient death in the first 90 days after ureteral reconstruction. Long-term patient and graft 


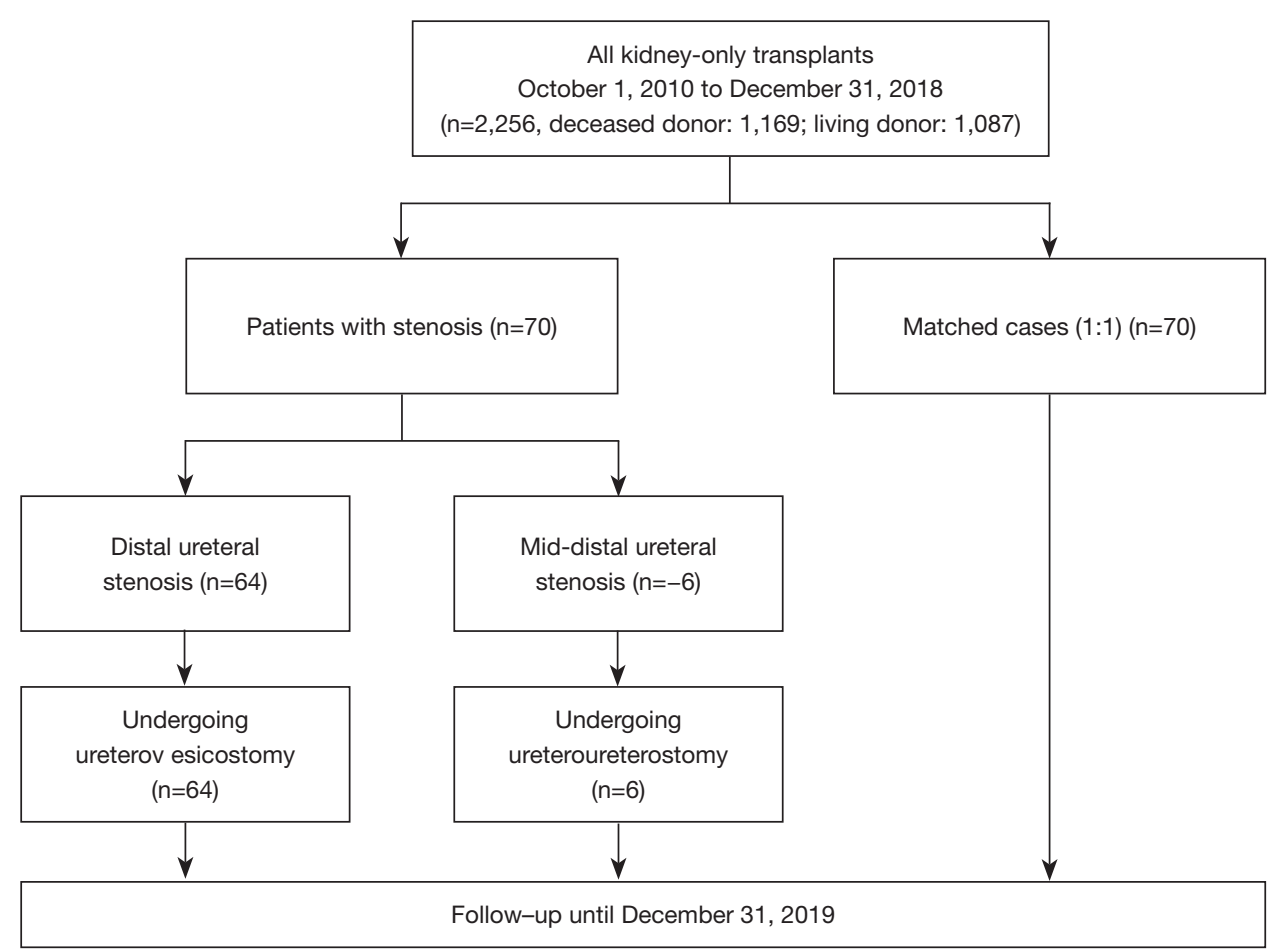

Figure 3 Outcome after treatment with open ureteral reconstruction surgery of ureteric stenosis in 70 renal allografts.

Table 3 The surgical complications according to Clavien-Dindo classification

\begin{tabular}{llll}
\hline Patient & The surgical complication & Intervention method & Clavien-Dindo classification \\
\hline NO. 5 & The renal vein injury & Repairing and hemodialysis & Grade III \\
NO. 19 & Subcapsular hematoma & Self absorption & Grade II \\
NO. 50 & Urinary leakage & Urine effusion drainage & Grade III \\
NO. 61 & Urinary leakage & Urine effusion drainage & Grade III \\
\hline
\end{tabular}

survivals in the stenosis group and matched control group were not significantly different (Figures 4,5). The survival rate of patient in stenosis group at 110 months was $98.6 \%$ (95\% CI: $99.8 \%, 90.3 \%$ ), in control group was $95.3 \%$ (95\% CI: 98.8\%, 81.8\%, P>0.05); the allograft survival rate was $90.4 \%$ (95\% CI: $96.6 \%, 74.4 \%)$ in stenosis group and 85.1\% (95\% CI: 95.2\%, 59.1\%) in controls ( $>>0.05)$.

Renal allograft loss occurred in 4 of 70 patients with ureteral stenosis. One patient died of pulmonary infection 6 months after ureteral reconstruction; two patients lost the renal allograft due to acute antibody-mediated rejection 1.5 and 3 years after ureteral reconstruction; one patient suffered graft failure due to acute cellular rejection 19 months after ureteral reconstruction, and the three survivors returned to dialysis. Four of the 70 patients lost their renal allograft in the control group. One patient died of pulmonary infection 3 years after transplant; one patient died of severe hepatitis 15 months after transplant; one patient lost the renal allograft due to acute antibody-mediated rejection 6 years after transplant; and one patient suffered graft failure due to acute cellular rejection 40 months after transplant. There was no significant difference in the rate of mortality and allograft losses between the 2 groups.

\section{Discussion}

To the best of our knowledge, this study has the largest sample size on open reconstruction under MRU localization 


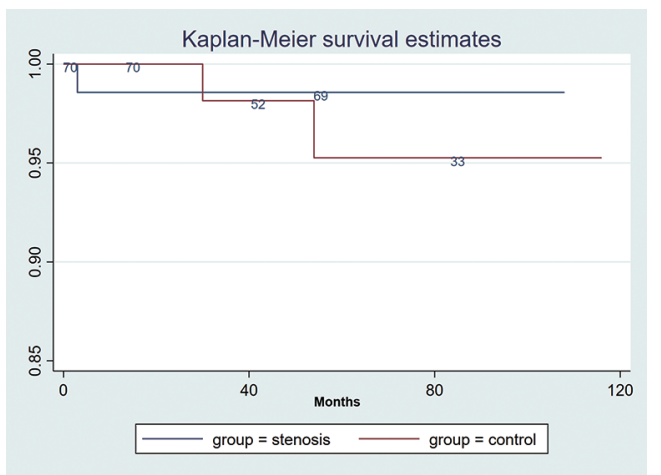

Figure 4 Age-censored Kaplan-Meier curves of patient survival in 70 patients with ureteric stenosis of renal allografts compared with a matched control group. 110-month patient survival: 98.6\% (95\% CI: $99.8 \%, 90.3 \%$ ) in stenosis group vs. $95.3 \%$ (95\% CI: $98.8 \%$, $81.8 \%$ ) in control group, $\mathrm{P}=0.758$; log-rank test.

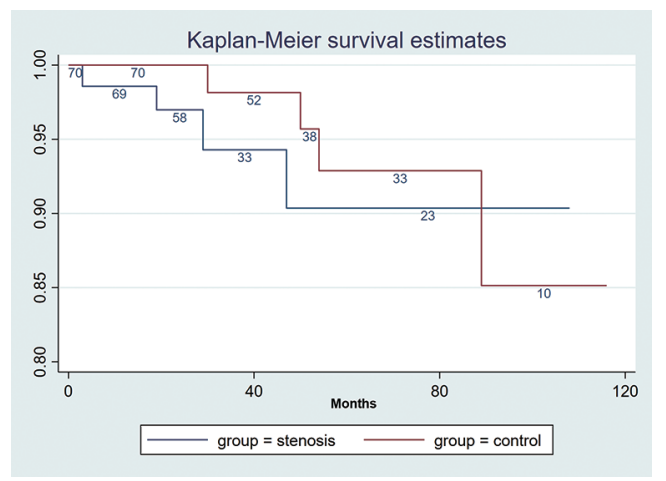

Figure 5 Age-censored Kaplan-Meier curves of graft survival in 70 patients with ureteric stenosis of renal allografts compared with a matched control group. 110-month graft survival: 90.4\% (95\% CI: $96.6 \%, 74.4 \%$ ) in stenosis group vs. $85.1 \%$ (95\% CI: $95.2 \%$, $59.1 \%$ ) in control group, $\mathrm{P}=0.546$; log-rank test.

for ureteral stenosis after renal transplantation. Open surgery under MRU localization was considered and offered to all recipients with ureteral stenosis in our study. The incision was one-third to half the length of the original incision. The ureteral obstruction site can be quickly located according to the preoperative MRU positioning. The overall success rate was $100 \%$ and the graft function was salvaged in all cases. There was no recurrence of stenosis after a mean follow-up of $38.9 \pm 26.3$ months. The complication rate was $5.7 \%$. All complications were controllable with conservative treatment, and no surgical intervention was needed. The results showed that the prognosis of the renal recipients with ureteral stenosis after ureteral reconstruction was similar to those without ureteral stenosis.

For the diagnosis of ureteral stricture, ultrasound first indicated hydronephrosis of the transplanted kidney, which was confirmed by MRU. In MRU imaging, narrow regions and lengths were clearly seen without the use of any contrast agent. The self-made and water-agent pill placed at the umbilicus was clearly displayed on the T2weighted image, which can help accurately positioning, so that ureteral obstruction segment would be precisely marked on the patient's body before surgery. The ureteral obstruction site can be quickly located according to the mark on the body surface during the operation. Six of 70 patients were confirmed to have mid-distal stenosis, and all of them underwent anastomosis with the native ureter during the operation, suggesting the reliability of MRU diagnosis. Computed tomography urography (CTU) can also clearly show the obstruction site, but CTU requires the use of contrast agents, which may cause kidney injury. The antegrade needle pyelogram and Whitaker test can also clearly show the obstruction site, however, this procedure requires a puncture of the kidney and the use of contrast agents, which are harmful to the kidney. The advantage of MRU is that MRU does not need contrast agents, while the images are equally good to CTU and Whitaker test.

The percutaneous endoscopic stenting, with or without dilatation, and surgical re-construction are the two most common therapeutic options currently employed to manage kidney recipients with ureteral strictures. Open surgery is associated with a greater morbidity, longer hospitalization, prolonged recovery and a higher risk for more serious complications, including graft loss and perioperative mortality compared with interventional treatment (9-11). The endoscopic techniques are more expensive but less invasive with lower rates of morbidity, and shorter hospital stay compared with open surgery. However, the successful rate of endoscopic treatment is lower and recurrence rate is higher compared with open surgery, sometimes multiple interventions are required or surgical treatment is needed in case of failure of interventional method. Moreover, stent implantation requires a regular stent replacement and it is prone to urinary tract infection (12-16). Kwong et al. reported that ureteral dilatation is the most common primary endoscopic treatment, with a success rate of $58.6 \%$ (95\% CI: 50.1-66.7, $\mathrm{n}=133$ ) (17). The range of restenosis rate in the literature is $10-45 \%(18-20)$. Patients with refractory ureteral stricture still need to be treated by open 
surgery after intervention failure. The result of our study showed that open reconstruction under MRU localization for ureteral stenosis after renal transplantation was safe and effective in the management of transplant ureteral stricture.

Although the result of our study was excellent, there are also shortcomings. The limitations of our study are the retrospective design and the long study period, which may have introduced unrecognized biases. We attempted to reduce such biases by matching for year of transplantation, but we cannot discount the possibility that biases may have been introduced by improved medical and surgical management. We only used two reconstruction techniques and Boari flap reconstruction was not normally carried out in our center (21).

\section{Conclusions}

MRU is an effective method for non-invasive and accurate diagnosis of ureteral stenosis in kidney transplant recipients. Open ureteral reconstruction surgery under MRU localization for treatment of ureter stenosis after kidney transplantation had a high success rate, low recurrence rate and high safety. This approach ensures long-term allograft and patient survivals, which is statistically comparable with those without the complication of ureteral stenosis. In general, our study suggests that kidney recipients with ureteral stenosis could be initially managed with open ureteral reconstruction under MRU localization before the innovation of minimally invasive technology.

\section{Acknowledgments}

We thank Dr. Hongqun Liu for language editing.

Funding: This work was supported by the national natural science foundation of China (grant number: 81970647).

\section{Footnote}

Reporting Checklist: The authors have completed the TREND reporting checklist. Available at http://dx.doi. org/10.21037/tau-20-1404

Data Sharing Statement: available at http://dx.doi. org/10.21037/tau-20-1404

Conflicts of interest: All authors have completed the ICMJE uniform disclosure form (available at http://dx.doi. org/10.21037/tau-20-1404). The authors have no conflicts of interest to declare.

Ethical Statement: The authors are accountable for all aspects of the work in ensuring that questions related to the accuracy or integrity of any part of the work are appropriately investigated and resolved. The study was conducted in accordance with the Declaration of Helsinki (as revised in 2013). The study was approved by the ethics committee of the First Affiliated Hospital, School of Medicine, Zhejiang University (NO. 2019-655: the registration number of ethics board) and individual consent for this retrospective analysis was waived.

Open Access Statement: This is an Open Access article distributed in accordance with the Creative Commons Attribution-NonCommercial-NoDerivs 4.0 International License (CC BY-NC-ND 4.0), which permits the noncommercial replication and distribution of the article with the strict proviso that no changes or edits are made and the original work is properly cited (including links to both the formal publication through the relevant DOI and the license). See: https://creativecommons.org/licenses/by-nc-nd/4.0/.

\section{References}

1. Hau HM, Tautenhahn HM, Schmelzle M, et al. Management of urologic complications in renal transplantation: a single-center experience. Transplant Proc 2014;46:1332-9.

2. Sandhu K, Masters J, Ehrlich Y. Ureteropyelostomy using the native ureter for the management of ureteric obstruction or symptomatic reflux following renal transplantation. Urology 2012;79:929-32.

3. Doehn C, Böse N, Meyer A, et al. Whose transplant function fails after ureteral revision following kidney transplantation? Transplant Proc 2010;42:1716-8.

4. Mundy AR, Podesta ML, Bewick M, et al. The urological complications of 1000 renal transplants. Br J Urol 1981;53:397-402.

5. Dreikorn K. Problems of the distal ureter in renal transplantation. Urol Int 1992;49:76-89.

6. Keller H, Nöldge G, Wilms H, et al. Incidence, diagnosis, and treatment of ureteric stenosis in 1298 renal transplant patients. Transpl Int 1994;7:253-57.

7. Shoskes DA, Hanbury D, Cranston D, et al. Urological complications in 1,000 consecutive renal transplant recipients. J Urol 1995;153:18-21.

8. Emiroglu R, Karakayall H, Sevmis S, et al. Urologic 
complications in 1275 consecutive renal transplantations. Transplant Proc 2001;33:2016-7.

9. Faenza A, Nardo B, Catena F, et al. Ureteral stenosis after kidney transplantation: interventional radiology or surgery? Transplant Proc 2001;33:2045-6.

10. Berli JU, Montgomery JR, Segev DL, et al. Surgical management of early and late ureteral complications after renal transplantation: techniques and outcomes. Clin Transplant 2015;29:26-33.

11. Pike TW, Pandanaboyana S, Hope-Johnson T, et al. Ureteric reconstruction for the management of transplant ureteric stricture: a decade of experience from a single centre. Transpl Int 2015;28:529-34.

12. Jalaeian H, Talaie R, Hunter DW, et al. Comparison of tandem ureteral stents, cryoplasty, and cutting balloon ureteroplasty in treatment of refractory transplant ureteral strictures. Clin Transplant 2020;34:e13859.

13. Juaneda B, Alcaraz A, Bujons A, et al. Endourological management is better in early-onset ureteral stenosis in kidney transplantation. Transplant Proc 2005;37:3825-7.

14. Collado A, Caparrós J, Guirado L, et al. Balloon dilatation in the treatment of ureteral stenosis in kidney transplant recipients. Eur Urol 1998;34:399-403.

15. Benoit G, Alexandre L, Moukarzel M, et al. Percutaneous anterograde dilatation of ureteral strictures in kidney

Cite this article as: Liu G, Wang X, Huang H, Wang R, Peng W, Chen J, Wu J. Perfect outcome of kidney recipients with ureteral stenosis after treatment with open surgery under magnetic resonance urography localization. Transl Androl Urol 2021;10(3):1160-1169. doi: 10.21037/tau-20-1404 transplants. Transplant Proc 1994;26:290-1.

16. Arpali E, Qaoud TA, Martinez E, et al. Impact of ureteral stricture and treatment choice on long-term graft survival in kidney transplantation. Am J Transplant 2018;18:1977-85.

17. Kwong J, Schiefer D, Aboalsamh G, et al. Optimal management of distal ureteric strictures following renal transplantation: a systematic review. Transpl Int 2016;29:579-88.

18. Aytekin C, Boyvat F, Harman A, et al. Percutaneous therapy of ureteral obstructions and leak after renal transplantation: long-term results. Cardiovasc Intervent Radiol 2007,30:1178-84.

19. Pappas P, Giannopoulos A, Stravodimos KG, et al. Obstructive uropathy in the transplanted kidney: definitive management with percutaneous nephrostomy and prolonged ureteral stenting. J Endourol 2001;15:719-23.

20. Yong AA, Ball ST, Pelling MX, et al. Management of ureteral strictures in renal transplants by antegrade balloon dilatation and temporary internal stenting. Cardiovasc Intervent Radiol 1999,22:385-8.

21. Charlesworth M, Marangoni G, Ahmad N. High ureteric injury following multiorgan recovery: successful kidney transplant with Boari flap ureterocystostomy reconstruction. Arab J Nephrol Transplant 2011;4:155-8. 